\title{
Computing Galois groups by means of Newton polygons
}

\author{
by \\ Michael Kölle and Peter Schmid (Tübingen)
}

Newton polygons are useful for computing decompositions of primes in extension rings, and for computing Galois groups. Suppose $f$ is a polynomial with coefficients in an algebraic number field $K$ and $\mathfrak{p}$ is a finite prime of $K$. Then, following Ore [10], one can associate to $f$ certain polynomials $f_{m} \in K[X]$ according to the slopes, $m$, of the sides of its Newton polygon with respect to $\mathfrak{p}$. Under some mild assumptions the Galois groups of the $f_{m}$, viewed as polynomials over the $\mathfrak{p}$-adic completion $K_{\mathfrak{p}}$, turn out to be constituents of $\mathrm{Gal}_{K_{\mathfrak{p}}}(f)$. The point is that the $f_{m}$ are usually much easier to handle than $f$, often they are pure polynomials.

1. Introduction. Originally Newton introduced polygons in order to investigate complex curves of two variables leading to what is now called the Puiseux series of a curve (cf. [1, pp. $494 \mathrm{ff}$.] for details). The method also applies to polynomials in one variable by looking at the various $g$-adic expansions. Such a theory has been developed by Ore [10] some eighty years ago. Ore's work has found recent interest (e.g. see [2], [5], [7], [8]). The objective of the present paper is to show how his ideas apply for computing Galois groups of (global) polynomials.

We fix an algebraic number field $K$, a finite prime $\mathfrak{p}$ of $K$, and a normalized polynomial $f \in K[X]$ of degree $n \geq 1$ :

$$
f=\sum_{i=0}^{n}(-1)^{i} a_{i} X^{n-i}=X^{n}-a_{1} X^{n-1}+\cdots+(-1)^{n} a_{n} .
$$

Here "normalized" means that $a_{0}=1$ and that $a_{n} \neq 0$. Denote by $v_{\mathfrak{p}}$ the (exponential) $\mathfrak{p}$-adic valuation of $K$. The (standard) Newton polygon of $f$ with respect to $\mathfrak{p}$ (and to $g(X)=X$ ) is the convex hull of the points $\left(i, v_{\mathfrak{p}}\left(a_{i}\right)\right)$, with $a_{i} \neq 0$, in the Euclidean $\mathbb{R}^{2}$. We pick a side $S_{m}$ of this polygon, determined by its slope $m$. Write $m=h / e$, where $h$ and $e$ are

2000 Mathematics Subject Classification: Primary 11S20; Secondary 11R32. 
relatively prime rational integers and $e>0$; this is made unique by letting $e=1$ in case $m=0$. Let $S_{m}$ begin with the point $\left(s, v_{\mathfrak{p}}\left(a_{s}\right)\right)$ and end with $\left(t, v_{\mathfrak{p}}\left(a_{t}\right)\right)$. Then there is a unique positive integer $d$ such that $d e=t-s$ is the length of $S_{m}$ and $d h=v_{\mathfrak{p}}\left(a_{s}^{-1} a_{t}\right)$ is its height.

Let us fix some further notation. Let $L$ be "the" splitting field of $f$ and $G=\operatorname{Gal}(L \mid K)$. We write $G=\operatorname{Gal}_{K}(f)$ when $G$ is understood as a permutation group on the set $Z_{f}$ of zeros of $f$. Let $\mathfrak{P}$ be a prime of $L$ above $\mathfrak{p}$ (unique up to $G$-conjugacy) and denote by $G_{\mathfrak{P}}$ and $I_{\mathfrak{P}}$ its decomposition and inertia groups, respectively. We extend $v_{\mathfrak{p}}$ uniquely to the $\mathfrak{p}$-adic completion $K_{\mathfrak{p}}$ and to some algebraic closure $\bar{K}_{\mathfrak{p}}$ containing $L_{\mathfrak{P}}$. Then $L_{\mathfrak{P}}=K_{\mathfrak{p}} L$ is the topological closure of $L$ in $\bar{K}_{\mathfrak{p}}$ and $G_{\mathfrak{P}}=\operatorname{Gal}\left(L_{\mathfrak{P}} \mid K_{\mathfrak{p}}\right)$ in the natural way. Let $k_{\mathfrak{p}}$ be the (finite) residue class field of $K_{\mathfrak{p}}$.

The rational numbers $m_{i}$ occurring as slopes of the sides of the Newton polygon of $f$ are characterized by the property that the sets $Z_{f, m_{i}}$ of roots of $f$ with $v_{\mathfrak{p}}$-value $m_{i}$ are not empty (see Statement 1 below). Moreover,

$$
Z_{f}=\biguplus_{i} Z_{f, m_{i}}
$$

is a decomposition into blocks of $G_{\mathfrak{P}}$. By its very definition the Newton polygon only can give information on $G_{\mathfrak{P}}$. Hence our target is to describe the constituent $G_{\mathfrak{P}}^{Z_{f, m}}$ (for the chosen slope $m$ ).

If $f$ is separable (nonzero discriminant) one knows that $\left|Z_{f, m}\right|=d e$ (Statement 1), and from a result of van der Waerden [11] one gets some information on the action of $G_{\mathfrak{P}}$ on $Z_{f, m}$ (see Statement 4). But this is helpful for computations only when knowing precisely the decomposition of $\mathfrak{p}$ in the root fields $K(\theta)$ for $\theta \in Z_{f, m}$. The basic idea is to replace $Z_{f, m}$ by the roots of a polynomial $f_{m}$ easily obtained from $S_{m}$. Following Ore [10] we introduce this factor $f_{m}$ and the polynomial $f_{S}$ associated to $S=S_{m}$. For each $i \geq 0$ we have $v_{\mathfrak{p}}\left(a_{s}^{-1} a_{s+i}\right) \geq i m$, and equality holds precisely when $\left(s+i, v_{\mathfrak{p}}\left(a_{s+i}\right)\right) \in S_{m}$. The factor is defined by

$$
f_{m}=\sum_{\substack{i=0 \\\left(s+i, v_{\mathfrak{p}}\left(a_{s+i}\right)\right) \in S_{m}}}^{d e}(-1)^{i} a_{s}^{-1} a_{s+i} X^{d e-i},
$$

ignoring the points not lying on $S_{m}$. The polynomial $f_{m} \in K[X]$ is normalized and its Newton polygon consists of one side, say $S$, with length de and slope $m$. Since $m=h / e$ and $(h, e)=1$ only points of the form $(j h, j e)$ arise from $f_{m}$ on $S(0 \leq j \leq d)$. So, fixing an element $\pi$ in $K$ of order 1 at $\mathfrak{p}\left(v_{\mathfrak{p}}(\pi)=1\right)$, we may "shorten" $f_{m}$ obtaining a normalized polynomial $f_{S} \in K[X]$ of degree $d$ such that $f_{m}$ is obtained from $f_{S}$ by substituting $X \mapsto \pi^{-h} X^{e}$ and multiplying the resulting polynomial with $\pi^{d h}$. 
The nonzero coefficients of $f_{S}$ are $\mathfrak{p}$-units. This $f_{S}$ as well as the reduction $\bar{f}_{S}=f_{S} \bmod \mathfrak{p}$ will be called the polynomial(s) associated to $S$ (or to $S_{m}$ ).

THEOREM. Suppose that $\mathfrak{p}$ does not divide the discriminant of $f_{S}$, that is, $\bar{f}_{S}=f_{S} \bmod \mathfrak{p}$ is separable. Then $f_{m}$ is separable and $\left|Z_{f, m}\right|=d e$ $\left(=\operatorname{deg}\left(f_{m}\right)=\right.$ length of the side $\left.S_{m}\right)$. If in addition $\mathfrak{p} \nmid e$, then the following hold:

(i) For every root $\beta$ of $f_{m}$ there exists $\theta \in Z_{f, m}$ such that $K_{\mathfrak{p}}(\beta)=$ $K_{\mathfrak{p}}(\theta)$, and vice versa.

(ii) The constituent $G_{\mathfrak{P}}^{Z_{f, m}}$ is permutation isomorphic to $\operatorname{Gal}_{K_{\mathfrak{p}}}\left(f_{m}\right)$.

(iii) $I_{\mathfrak{P}}^{Z_{f, m}}$ is cyclic generated by an element which is the product of $d$ disjoint e-cycles on $Z_{f, m}$.

(iv) $G_{\mathfrak{P}}^{Z_{f, m}} / I_{\mathfrak{P}}^{Z_{f, m}}$ is, as a permutation group on the set of orbits of $I_{\mathfrak{P}}$ on $Z_{f, m}$, permutation isomorphic to $\mathrm{Gal}_{k_{\mathfrak{p}}}\left(\bar{f}_{S}\right)$.

Following Ore [10] we say that the side $S_{m}$ is regular provided $\bar{f}_{S}$ is separable. This, as well as possible factorizations of $\bar{f}_{S}$, are (essentially) independent of the choice of the prime element $\pi$ in the definition of $f_{S}$. For altering $\pi$ amounts to a substitution $X \mapsto u X$ in $f_{S}(X)$ for some $\mathfrak{p}$-unit $u \in K$, followed by multiplication with $u^{-d}$. If $S_{m}$ is not regular, then there is a normalized $\mathfrak{p}$-integral polynomial $g \in K[X]$ whose reduction $\bmod \mathfrak{p}$ is irreducible and a multiple divisor of $f_{S} \bmod \mathfrak{p}(g \nmid f)$. Then one should examine the $g$-adic Newton polygon of $f$ with respect to $\mathfrak{p}$ (see Section 7 below).

Let us describe two interesting special cases of the Theorem:

Corollary 1. Suppose that the length of $S_{m}$ divides its height. If $\bar{f}_{S}=$ $f_{S} \bmod \mathfrak{p}$ is a product of pairwise distinct normalized irreducible polynomials in $k_{\mathfrak{p}}[X]$ of degrees $d_{i}$, then $G_{\mathfrak{P}}^{Z_{f, m}}$ is generated by a permutation which is the product of the corresponding disjoint $d_{i}$-cycles.

Here $e=1$. By assumption $S_{m}$ is regular. By statement (iii) of the Theorem $I_{\mathfrak{P}}^{Z} Z_{f, m}=1$ and so

$$
G_{\mathfrak{P}}^{Z_{f, m}} \cong \mathrm{Gal}_{k_{\mathfrak{p}}}\left(\bar{f}_{S}\right)
$$

by statement (iv). This yields the corollary, which generalizes a classical result due to Dedekind and Bauer (e.g. see Matzat [6, p. 127]).

Corollary 2. Suppose that length and height of $S_{m}$ are nonzero and relatively prime. If $\mathfrak{p}$ does not divide e, then $G_{\mathfrak{P}}^{Z_{f, m}}=I_{\mathfrak{P}}^{Z_{f, m}}$ is cyclic of order $e$.

Here $d=1$. It follows that $f_{m}=X^{e}-b$ is a pure polynomial and that $f_{S}=X-\pi^{-h} b$ is linear, where $b=(-1)^{e+1} a_{s}^{-1} a_{s+e}$ and $\pi^{-h} b$ is a $\mathfrak{p}$-unit 
in $K$. The Theorem applies. Note also that $f_{m}$ must be irreducible over $K_{\mathfrak{p}}$ and that its splitting field agrees with that of a certain prime factor of $f$ over $K_{\mathfrak{p}}$.

The assumption $\mathfrak{p} \nmid e$ refers to tame ramification. The Theorem indeed implies the familiar result on totally and tamely ramified local extensions (see [9, II.7.7]). In this case $f$ is an Eisenstein polynomial with respect to $\mathfrak{p}$, the Newton polygon of $f$ consisting of one side $S=S_{m}$ with slope $m=1 / n$. Here we have $f_{m}=X^{n}-(-1)^{n+1} a_{n}$ and $f_{S}=X-u$ for some $\mathfrak{p}$ unit $u$. If $\mathfrak{p}$ does not divide $e=n$ (tame ramification) then, by the Theorem, $K_{\mathfrak{p}}(\theta)=K_{\mathfrak{p}}\left(\sqrt[n]{(-1)^{n+1} a_{n}}\right)$ for a root $\theta$ of $f$ (suitably chosen).

Our terminology follows Neukirch [9] (number theory) and Dixon-Mortimer [3] (permutation groups).

2. Background. We keep the assumptions and notations introduced above. Thus $S_{m}$ is a side of the Newton polygon of $f$ with respect to $\mathfrak{p}$ which has length de and slope $m=h / e$, with the extreme points $\left(s, v_{\mathfrak{p}}\left(a_{s}\right)\right)$ and $\left(t, v_{\mathfrak{p}}\left(a_{t}\right)\right), s<t(t-s=d e)$.

StATEMENT 1. Let the roots $\theta_{1}, \ldots, \theta_{n}$ of $f$ (counting multiplicities) be indexed in such a way that $v_{\mathfrak{p}}\left(\theta_{i}\right) \leq v_{\mathfrak{p}}\left(\theta_{j}\right)$ if $i<j$. Then $\theta_{s+1}, \ldots, \theta_{t}=\theta_{s+d e}$ are just those roots with $v_{\mathfrak{p}}$-value $m$, and the polynomial $\widehat{f}_{m}=\prod_{i=1}^{d e}\left(X-\theta_{s+i}\right)$ has its coefficients in $K_{\mathfrak{p}}$.

For a proof we refer to [9, Theorems II.6.3 and II.6.4]. We regard $\widehat{f}_{m} \in$ $K_{\mathfrak{p}}[X]$ as a local polynomial (though its coefficients are in $\left.K_{\mathfrak{p}} \cap L\right)$. We have $\left|Z_{f, m}\right| \leq d e$ and equality holds if and only if $\widehat{f}_{m}$ is separable.

Recall that $\left|I_{\mathfrak{P}}\right|=e_{\mathfrak{P}}$ is the ramification index of $\mathfrak{P}$ over $\mathfrak{p}([9$, II.9.9]). Since $v_{\mathfrak{P}}\left(\theta_{t}\right)=e_{\mathfrak{P}} v_{\mathfrak{p}}\left(\theta_{t}\right)=h \cdot e_{\mathfrak{P}} / e$ is an integer, we see that $e$ is a divisor of $\left|I_{\mathfrak{P}}\right|$. This is a basic, and often used, information on the order of the Galois group $G$ which is immediate from the Newton polygon.

StATEMEnt 2. Let $f=\prod_{i} f^{(i)}$ be a factorization over $K$ into normalized polynomials $f^{(i)}$. Then $S_{m}$ is obtained by "joining" the sides $S_{m}^{(j)}$ of the Newton polygons of those $f^{(j)}$ admitting the slope $m$. Moreover, $f_{m}$ and $\prod_{j} f_{m}^{(j)}$ give rise to the same points on $S_{m}$ and $f_{S} \equiv \prod_{j} f_{S}^{(j)}(\bmod \mathfrak{p})$.

For $\mathfrak{p}$-integral polynomials (and $m>0$ ) this follows from the results proved in Chapter 1 of [10] (see Theorems 5 and 7). That it is true in general may be seen from Statement 1 on the basis of Proposition 1 below. Of course, to a normalized polynomial $\widehat{f} \in K_{\mathfrak{p}}[X]$ we assign the obvious Newton polygon (with respect to $v_{\mathfrak{p}}$ ). Statement 2 carries over to such local polynomials. If the polygon of $\widehat{f}$ consists of one side $\widehat{S}$ then, ignoring the coefficients of the polynomial yielding points not lying on $\widehat{S}$ and shortening 
the resulting factor, picking the same prime element $\pi \in K$ as before, we obtain an associated (local) polynomial $\widehat{f}_{\widehat{S}}$.

Statement 3. Suppose $\widehat{f} \in K_{\mathfrak{p}}[X]$ is normalized, irreducible and $\widehat{N}=$ $K_{\mathfrak{p}}(\theta)$ for some root $\theta$ of $\widehat{f}$. Then the Newton polygon of $\widehat{f}$ consists of one side $\widehat{S}$ and the associated polynomial $\widehat{f}_{\widehat{S}} \bmod \mathfrak{p}$ is a power of an irreducible polynomial $\bar{g} \in k_{\mathfrak{p}}[X]$. The degree of $\bar{g}$ divides the residue class degree $r\left(\widehat{N} \mid K_{\mathfrak{p}}\right)$.

From Statement 1 it is immediate that the Newton polygon is as asserted (see also [9, II.6.5]). By abuse of notation let $\widehat{f}=f$ and $\widehat{S}=S_{m}=S$ (so that $\operatorname{deg}(\widehat{f})=n=d e$ and $m=h / e)$. So by definition

$$
f_{m}(X)=\pi^{d h} f_{S}\left(\pi^{-h} X^{e}\right) .
$$

If $m=0$ then $f$ and $f_{m}=f_{S}$ are integral and have the same reduction mod $\mathfrak{p}$, which must be a power of an irreducible $\bar{g}$ by Hensel's lemma ([9, II.4.6]). Then the image of $\theta$ in the residue class field of $\widehat{N}$ is a root of $\bar{g}$. It follows that $\operatorname{deg}(\bar{g})$ divides $r\left(\widehat{N} \mid K_{\mathfrak{p}}\right)$. Recall that the maximal unramified subextension of $\widehat{N} \mid K_{\mathfrak{p}}$ is cyclic of degree $r\left(\widehat{N} \mid K_{\mathfrak{p}}\right)$. (Our notation differs from that in $[9]$, reserving the letter $f$ for polynomials.)

The general case is treated as follows (cf. [10, Theorem 3 in Chap. 2] for the case $m>0)$. We know from Statement 1 that $v_{\mathfrak{p}}\left(\pi^{-h} \theta^{e}\right)=-h+e m=0$. Hence $\pi^{-h} \theta^{e}$ is a unit in $\widehat{N}$. Let $\bar{g}$ be the minimum polynomial over $k_{\mathfrak{p}}$ of its residue class. Then $\operatorname{deg}(\bar{g})$ divides $r\left(\widehat{N} \mid K_{\mathfrak{p}}\right)$. Let $g$ be the minimum polynomial over $K_{\mathfrak{p}}$ of $\pi^{-h} \theta^{e}$. Then $g$ is integral and the reduction $g \bmod \mathfrak{p}$ a power of $\bar{g}$ (Hensel). Let $t=\operatorname{deg}(g)$. Define the polynomial $\widetilde{g}$ by

$$
\widetilde{g}(X)=\pi^{t h} g\left(\pi^{-h} X^{e}\right) .
$$

Observe that $g$ and $\widetilde{g}$ are normalized. We even know that $g(0)$ is a unit in $K_{\mathfrak{p}}$. Using the fact that $g$ is integral this shows that the Newton polygon of $\widetilde{g}$ is a side $\widetilde{S}$ with length te and slope $m$. Its associated polynomial $\widetilde{g}_{\widetilde{S}}$ is obtained from $g$ by leaving out those monomials where the coefficients are nonunits. In particular, it has the same reduction $\bmod \mathfrak{p}$. Now $\theta$ is a root of $\widetilde{g}$ and so $\widetilde{g}=f \cdot \widetilde{f}$ for some $\widetilde{f} \in K_{\mathfrak{p}}[X]$, because $f$ is the minimum polynomial of $\theta$. It follows from Statement 2 that $f_{S} \bmod \mathfrak{p}\left(\right.$ like $\left.\widetilde{g}_{\widetilde{S}} \bmod \mathfrak{p}\right)$ is a power of $\bar{g}$, as desired.

The local polynomial $\widehat{f}_{m}$ of Statement 1 is normalized. By considering the elementary symmetric functions of its roots we see that its polygon consists of one side of length de and slope $m$ which, therefore, may be identified with the side $S$ assigned to the global polynomial $f_{m}$. In what follows we write $\widehat{f}_{S}$ in place of $\left(\widehat{f}_{m}\right)_{S}$ for the associated polynomial. 
Statement 4. Suppose $f$ is irreducible over $K$ and $N=K(\theta)(\subseteq L)$ for some root $\theta$ of $f$. Let $\mathcal{M}$ denote the set of primes $\wp$ of $N$ above $\mathfrak{p}$ satisfying $v_{\wp}(\theta)=e_{\wp} m$, where $e_{\wp}$ is the ramification index over $\mathfrak{p}$. Then there are normalized irreducible polynomials $\widehat{f}_{\wp} \in K_{\mathfrak{p}}[X]$ of degree $e_{\wp} r_{\wp}$, with $r_{\wp}$ denoting the residue class degree, such that

$$
\widehat{f}_{m}=\prod_{\wp \in \mathcal{M}} \widehat{f}_{\wp} .
$$

Over the unramified extension field of $K_{\mathfrak{p}}$ of degree $r_{\wp}$ the polynomial $\widehat{f}_{\wp}$ decomposes into a product of Galois conjugate irreducible polynomials of degree $e_{\wp}(\wp \in \mathcal{M})$.

This follows from a classical result of van der Waerden [11]. In fact, the roots of $\widehat{f}_{\wp}$ belong to an orbit of $Z_{f}$ under the action of $G_{\mathfrak{P}}$, which in turn decomposes under $I_{\mathfrak{P}}$ into $r_{\wp}$ orbits of length $e_{\wp}$ (see also [6, Theorem 1 , p. 126]). The 1-1 correspondence $\wp \leftrightarrow \widehat{f}_{\wp}$ is achieved as follows: A double coset $G_{\theta} \sigma G_{\mathfrak{P}}$ for $\sigma \in G$ corresponds to the $G_{\mathfrak{P}}$-orbit of $\theta^{\sigma}$ and determines the prime $\wp=\mathfrak{P}^{\sigma^{-1}} \cap N$ (so that the $\mathfrak{P}^{(\tau \sigma \gamma)^{-1}}$ for $\tau \in G_{\theta}=\operatorname{Gal}(L \mid N)$, $\gamma \in G_{\mathfrak{P}}$, are all the primes in $L$ above $\left.\wp\right)$.

We have $v_{\wp}(\theta)=v_{\wp}\left(\theta^{\sigma}\right)$ for all primes $\wp$ of $N$ above $\mathfrak{p}$ and all $\sigma \in G$. It follows that $\wp \in \mathcal{M}$ if and only if there is $\sigma \in G$ such that $\mathfrak{P} \mid \wp^{\sigma}$ and $\theta^{\sigma} \in Z_{f, m}$, which gives the statement.

The primes $\wp$ in $\mathcal{M}=\mathcal{M}_{\theta}$ are said to belong to the side $S_{m}$. Since $v_{\wp}(\theta)=h \cdot e_{\wp} / e$ is an integer, $e$ divides $e_{\wp}$.

3. Global and local polynomials to a side. By definition the local polynomial $\widehat{f}_{m}$ is a divisor of $f$ over $K_{\mathfrak{p}}$ whereas the global polynomial $f_{m}$ is just constructed using certain coefficients of $f$. Computation of $\widehat{f}_{m}$ from $f$ is not easy and often appears even impracticable.

Lemma. Write $\widehat{f}_{m}=\sum_{i=0}^{d e}(-1)^{i} c_{i} X^{d e-i}$ (so that $\left.c_{0}=1\right)$. We have

$$
v_{\mathfrak{p}}\left(a_{s}^{-1} a_{s+i}-c_{i}\right)>i m \text { for all } i=0, \ldots, d e=t-s .
$$

Proof. We order the roots $\theta_{1}, \ldots, \theta_{n}$ of $f$ totally as in Statement 1. Then we have $v_{\mathfrak{p}}\left(\theta_{j}\right)<m$ for $j \leq s$ and $v_{\mathfrak{p}}\left(\theta_{j}\right)>m$ for $j>t=s+d e$. The coefficients $a_{i}$ of $f$ for $i \neq 0$ are the elementary symmetric functions of the $\theta_{j}$. We have $v_{\mathfrak{p}}\left(a_{i}\right)=v_{\mathfrak{p}}\left(\theta_{1} \cdots \theta_{i}\right)$ whenever $\left(i, v_{\mathfrak{p}}\left(a_{i}\right)\right)$ is an extreme point of the polygon. This holds, in particular, for $i=s$ (with the obvious convention when $s=0$ ). The coefficients $c_{i}, i \neq 0$, of $\widehat{f}_{m}$ are the elementary symmetric functions of the $\theta_{s+1}, \ldots, \theta_{t}$ (Statement 1 ). Hence

$$
c_{i}=\sum_{s<l_{1}<\cdots<l_{i} \leq t} \theta_{l_{1}} \cdots \theta_{l_{i}}
$$


for each $i=1, \ldots, d e$. It follows that $v_{\mathfrak{p}}\left(c_{i}\right) \geq i m$. Consider

$$
\varrho_{i}=a_{s+i}-a_{s} c_{i}=\sum_{1 \leq l_{1}<\cdots<l_{s+i} \leq n} \theta_{l_{1}} \cdots \theta_{l_{s+i}}-\left(\sum_{1 \leq l_{1}<\cdots<l_{s} \leq n} \theta_{l_{1}} \cdots \theta_{l_{s}}\right) c_{i} .
$$

Taking into account the expression for $c_{i}$, this $\varrho_{i}$ is the difference of terms where each term is a sum of products of just $s+i$ of the roots $\theta_{j}$ (allowing certain multiplicities). The partial sum

$$
\left(\theta_{1} \cdots \theta_{s}\right) \cdot \sum_{s<l_{1}<\cdots<l_{i} \leq t} \theta_{l_{1}} \cdots \theta_{l_{i}}
$$

occurs in both $a_{s+i}$ and $a_{s} c_{i}$, hence disappears in $\varrho_{i}$. But the $v_{\mathfrak{p}}$-value of this part is at least equal to $v_{\mathfrak{p}}\left(a_{s}\right)+i m$, and all other products appearing in $\varrho_{i}$ have larger $v_{\mathfrak{p}}$-values. We conclude that $v_{\mathfrak{p}}\left(\varrho_{i}\right)>v_{\mathfrak{p}}\left(a_{s}\right)+i m$. The result follows.

Proposition 1. The points lying on $S$ resulting from $f_{m}$ and from $\widehat{f}_{m}$ are the same and $f_{S} \equiv \widehat{f}_{S}(\bmod \mathfrak{p})$.

Proof. It follows from the lemma that both polynomials $f_{m}$ and $\widehat{f}_{m}$ yield the same points lying on $S$. Recall that $\widehat{f}_{S}=\left(\widehat{f}_{m}\right)_{S}$ is obtained from (the factor to) $\widehat{f}_{m}$, like $f_{S}$ from $f_{m}$, using the same prime element $\pi$. Thus the lemma even tells us that $f_{S}-\widehat{f}_{S}$ is divisible by $\pi$. The proof is complete.

4. Regularity. In this section we assume that $S_{m}$ is regular. Our discussion is motivated by the main theorem in Ore [10] (Theorem 5 in Chap. 2; see [2] and [7] for re-statements).

Proposition 2. Suppose $\mathfrak{p}$ does not divide the discriminant of $f_{S}$. Then both $\widehat{f}_{m}$ and $f_{m}$ are separable (of degree de). There is a 1-1 correspondence $\varphi \leftrightarrow \psi$ between the normalized prime factors over $K_{\mathfrak{p}}$ of $f_{m}$ and $\widehat{f}_{m}$ such that:

(i) Both $\varphi$ and $\psi$ have the same Newton polygon (a side with slope $m$ ) and the same associated polynomial $\bar{g} \in k_{\mathfrak{p}}[X]$, which is irreducible over $k_{\mathfrak{p}}$.

(ii) Letting $\theta$ and $\beta$ denote roots of $\varphi$ and $\psi$, respectively, both $K_{\mathfrak{p}}(\theta)$ and $K_{\mathfrak{p}}(\beta)$ have over $K_{\mathfrak{p}}$ ramification index e and residue class degree $\operatorname{deg}(\bar{g})$.

Proof. By hypothesis and Proposition 1 we may write

$$
\widehat{f}_{S} \bmod \mathfrak{p}=f_{S} \bmod \mathfrak{p}=\prod_{i} \bar{g}_{i},
$$

where the $\bar{g}_{i}$ are distinct normalized irreducible polynomials over $k_{\mathfrak{p}}$. Consider the normalized prime factors $f^{(j)}$ of $f$ over $K$ whose Newton polygon with respect to $\mathfrak{p}$ has a side $S_{m}^{(j)}$ with slope $m$. From Statements 1 and 2 
it follows that each $f^{(j)}$ is a simple divisor of $f$ and that $\widehat{f}_{m}=\prod_{j} \widehat{f}_{m}^{(j)}$ is separable of degree de.

Even more is true. By Statement 2 the associated polynomial mod $\mathfrak{p}$ to $f^{(j)}$ and $S_{m}^{(j)}$ is a product $\widetilde{g}_{j}$ of certain of the $\bar{g}_{i}$, and $\prod_{j} \widetilde{g}_{j}=f_{S} \bmod \mathfrak{p}$.

By Hensel's lemma we may write $f_{S}=\prod_{i} g_{i}$ where each $g_{i} \in K_{\mathfrak{p}}[X]$ is normalized, integral with reduction $\bar{g}_{i}$. Via the substitution $X \mapsto \pi^{-h} X^{e}$, followed by multiplication with $\pi^{\operatorname{deg}\left(g_{i}\right) h}$, from $g_{i}$ we get a normalized polynomial $f_{m}^{(i)} \in K_{\mathfrak{p}}[X]$ of degree $e \cdot \operatorname{deg}\left(g_{i}\right)$. Since $\sum_{i} \operatorname{deg}\left(g_{i}\right)=d=\operatorname{deg}\left(f_{S}\right)$ this yields a factorization

$$
f_{m}=\prod_{i} f_{m}^{(i)}
$$

over $K_{\mathfrak{p}}$. The Newton polygon of each $f_{m}^{(i)}$ must be a side with slope $m$ and with associated polynomial $g_{i}$. Since $\bar{g}_{i}$ is irreducible over $k_{\mathfrak{p}}$, from Statement 2 it follows that $f_{m}^{(i)}$ must be irreducible over $K_{\mathfrak{p}}$. Separability of $f_{S} \bmod \mathfrak{p}$ implies that $f_{m}$ is separable.

Replacing $f$ by some $f^{(j)}$, if necessary, we therefore may assume that $f$ is irreducible over $K$. Then, if we let $N=K(\theta)$ for some root $\theta$ of $f$, Statement 4 applies. We obtain the prime factorization

$$
\widehat{f}_{m}=\prod_{\wp \in \mathcal{M}} \widehat{f}_{\wp}
$$

over $K_{\mathfrak{p}}$ indexed by the set $\mathcal{M}$ of primes of $N$ belonging to $S_{m}$. By Statements 3 and 2 (and separability of $\widehat{f}_{S} \bmod \mathfrak{p}$ ) the Newton polygon of any $\widehat{f}_{\wp}$ is a side with slope $m$ and irreducible associated polynomial $\bar{g}_{\wp} \bmod \mathfrak{p}$, which must be one of the $\bar{g}_{i}$. In this manner we get a 1-1 correspondence $\wp \leftrightarrow i$ which we use to re-write $\bar{g}_{\wp}=\bar{g}_{i}$ and $f_{\wp}=f_{m}^{(i)}$. Thus $f_{m}=\prod_{\wp \in \mathcal{M}} f_{\wp}$ likewise.

Recall that $\operatorname{deg}\left(f_{\wp}\right)=e \cdot \operatorname{deg}\left(\bar{g}_{\wp}\right)$ and $\operatorname{deg}\left(\widehat{f}_{\wp}\right)=e_{\wp} \cdot r_{\wp}$ (Statement 4$)$, and that $e$ is a divisor of $e_{\wp}$ for all $\wp \in \mathcal{M}$. From Statement 3 we know that $\operatorname{deg}\left(\bar{g}_{\wp}\right)$ divides $r\left(\widehat{N} \mid K_{\mathfrak{p}}\right)$ where $\widehat{N}=K_{\mathfrak{p}}\left(\theta^{\sigma}\right)$ for some root $\theta^{\sigma}$ of $\widehat{f}_{\wp}$ in $L_{\mathfrak{P}}$ (with $\sigma \in G$ ). Then $\mathfrak{P} \mid \wp^{\sigma}$ and $\widehat{N}=\left(N^{\sigma}\right)_{\wp^{\sigma}}$. It follows that $r\left(\widehat{N} \mid K_{\mathfrak{p}}\right)=$ $r_{\wp}\left(=r_{\wp}\right)$. From

$$
\sum_{\wp} e \cdot \operatorname{deg}\left(\bar{g}_{\wp}\right)=\operatorname{deg}\left(f_{m}\right)=\operatorname{deg}\left(\widehat{f}_{m}\right)=\sum_{\wp} e_{\wp} \cdot r_{\wp}
$$

we may conclude that $e_{\wp}=e$ and $\operatorname{deg}\left(\bar{g}_{\wp}\right)=r_{\wp}$ for all $\wp \in \mathcal{M}$. This completes the proof.

5. Tame ramification. Let $\varphi$ and $\psi$ be normalized, irreducible polynomials of the same degree over $K_{\mathfrak{p}}$ having the same Newton polygon, a side $\Sigma$ with slope $m$ (with $m=h / e$ as usual). 
Proposition 3. Assume that $\varphi$ and $\psi$ have the same associated polynomial $\bar{g}$ and that it is irreducible over $k_{\mathfrak{p}}$. If $\mathfrak{p}$ does not divide e, then to every root $\theta$ of $\varphi$ there is a root $\beta$ of $\psi$ such that $K_{\mathfrak{p}}(\theta)=K_{\mathfrak{p}}(\beta)$, and vice versa.

Proof. By symmetry it suffices to show that, for a fixed root $\theta$ of $\varphi$, there exists a root $\beta$ of $\psi$ with the required property. In place of $v_{\mathfrak{p}}$ we use a multiplicative (discrete) valuation $|\cdot|$ on $\bar{K}_{\mathfrak{p}}$, somehow normalized $(|\pi|<1)$.

By Statement 1 all the roots of $\varphi$ and of $\psi$ have $v_{\mathfrak{p}}$-value $m=h / e$. Hence $|\theta|^{e}=|\pi|^{h}=|\beta|^{e}$ for each root $\beta$ of $\psi$. It follows that

$$
\theta^{e}=\pi^{h} u_{\theta}, \quad \beta^{e}=\pi^{h} u_{\beta}
$$

for some units $u_{\theta}, u_{\beta}\left(\left|u_{\theta}\right|=\left|u_{\beta}\right|=1\right)$.

Let $\widehat{T}$ be the unramified (cyclic) extension field of $K_{\mathfrak{p}}$ of degree $\operatorname{deg}(\bar{g})$ (within $\bar{K}_{\mathfrak{p}}$ ). Note that $\pi$ is a prime element of $\widehat{T}$. Over the residue class field of $\widehat{T}$ the polynomial $\bar{g}$ decomposes into a product of Galois conjugate linear polynomials, and $\varphi$ and $\psi$ decompose correspondingly over $\widehat{T}$ (in view of Statement 3). Replace $\varphi$ by the minimum polynomial of $\theta$ over $\widehat{T}$, and replace $\psi$ by the appropriate prime factor over $\widehat{T}$. The polynomials thus obtained will have the same Newton polygon, consisting of one side with slope $m$, length $e$ and height $h$, and with the same associated (linear) polynomial $\bmod \mathfrak{p}$ (Statement 2$)$.

Since $K_{\mathfrak{p}}(\theta)=\widehat{T}(\theta)$ and $K_{\mathfrak{p}}(\beta)=\widehat{T}(\beta)$ for every root $\beta$ of $\psi$, without loss of generality we may assume that $K_{\mathfrak{p}}=\widehat{T}$. Then $K_{\mathfrak{p}}(\theta)$ is a totally (and tamely) ramified extension of $K_{\mathfrak{p}}$ of degree $e=\operatorname{deg}(\varphi)$. A similar statement holds for $K_{\mathfrak{p}}(\beta)$ and any root $\beta$ of $\psi$. The polynomial $\bar{g}$ being linear (with $\bar{g}(0) \neq 0)$ the factors of $\varphi$ and $\psi$ for the side $\Sigma$ are pure polynomials

$$
X^{e}-\pi^{h} u, \quad X^{e}-\pi^{h} \widehat{u},
$$

respectively, where $u$ and $\widehat{u}$ are units in $K_{\mathfrak{p}}$ satisfying $u \equiv \widehat{u}(\bmod \mathfrak{p})$. Thus $|u-\widehat{u}|<1$. Clearly we may assume that $e>1(m \neq 0)$.

Suppose $\psi=X^{e}+c_{1} X^{e-1}+\cdots+c_{e-1} X+c_{e}$. Then $c_{e}=-\pi^{h} \widehat{u}$ and $v_{\mathfrak{p}}\left(c_{i}\right)>i m$ for all $i=1, \ldots, e-1$. Hence if $\beta$ is a root of $\psi$ then

$$
\left|c_{i} \beta^{e-i}\right|=\left|c_{i}\right| \cdot|\pi|^{(h / e) \cdot(e-i)}<|\pi|^{h}
$$

for $i=1, \ldots, e-1$. It follows that

$$
\left|\beta^{e}-\pi^{h} \widehat{u}\right|=\left|c_{1} \beta^{e-1}+\cdots+c_{e-1} \beta\right|<|\pi|^{h} .
$$

Thus $\left|u_{\beta}-\widehat{u}\right|<1$ for each root $\beta$ of $\psi$. By considering $\varphi$ in place of $\psi$ we get $\left|u_{\theta}-u\right|<1$ analogously. We infer that

$$
\left|u_{\theta}-\widehat{u}\right| \leq \max \left\{\left|u_{\theta}-u\right|,|u-\widehat{u}|,\left|\widehat{u}-u_{\beta}\right|\right\}<1 \text {. }
$$


This yields $\left|\theta^{e}-\pi^{h} \widehat{u}\right|=|\pi|^{h}\left|u_{\theta}-\widehat{u}\right|<|\pi|^{h}$ and so

$$
\begin{aligned}
|\psi(\theta)| & =\left|\left(\theta^{e}-\pi^{h} \widehat{u}\right)+\left(c_{1} \theta^{e-1}+\cdots+c_{e-1} \theta\right)\right| \\
& \leq \max \left\{\left|\theta^{e}-\pi^{h} \widehat{u}\right|, \max _{1 \leq k \leq e-1}\left|c_{k} \theta^{e-k}\right|\right\}<|\pi|^{h} .
\end{aligned}
$$

Suppose $\beta_{1}, \ldots, \beta_{e}$ are the roots of $\psi$. We have shown that

$$
\prod_{j=1}^{e}\left|\theta-\beta_{j}\right|=|\psi(\theta)|<|\pi|^{h} .
$$

Since $\left|\theta-\beta_{j}\right| \leq \max \left\{|\theta|,\left|\beta_{j}\right|\right\}=|\pi|^{h / e}$ for all $j$, there must be some root, say $\beta=\beta_{1}$, such that $|\theta-\beta|<|\pi|^{h / e}$. We assert that $K_{\mathfrak{p}}(\beta)=K_{\mathfrak{p}}(\theta)$ for this choice of $\beta$.

We consider the derivative of $\psi$ at $\beta$. Since $\mathfrak{p}$ does not divide $e$ by hypothesis, we have $|e|=1$. We obtain

$$
\left|\psi^{\prime}(\beta)\right|=\frac{1}{|\beta|} \cdot\left|e \beta^{e}+(e-1) c_{1} \beta^{e-1}+\cdots+c_{e-1} \beta\right|=|\beta|^{e-1}=|\pi|^{(e-1) \cdot h / e} .
$$

On the other hand, $\left|\psi^{\prime}(\beta)\right|=\prod_{j=2}^{e}\left|\beta-\beta_{j}\right|$ and $\left|\beta-\beta_{j}\right| \leq|\pi|^{h / e}$ for each $j$. Consequently, $\left|\beta-\beta_{j}\right|=|\pi|^{h / e}$ for all $j=2, \ldots, e$. But this implies that $|\theta-\beta|<\left|\beta-\beta_{j}\right|$ for all $j \neq 1$. By the lemma of Krasner ([9, p. 159]) this gives $K_{\mathfrak{p}}(\beta) \subseteq K_{\mathfrak{p}}(\theta)$. We must have equality since $\varphi$ and $\psi$ are irreducible over $K_{\mathfrak{p}}$ of the same degree.

6. The Galois group for a regular side. We are going to prove the Theorem stated in the introduction. Suppose $S_{m}$ is regular. Without assuming that $S_{m}$ is tame $(\mathfrak{p} \nmid e)$ we can describe the constituent $I_{\mathfrak{P}}^{Z}$, and the quotient $G_{\mathfrak{P}}^{Z_{f, m}} / I_{\mathfrak{P}}^{Z_{f, m}}$ as follows.

Proposition 4. Suppose $\bar{f}_{S}=f_{S} \bmod \mathfrak{p}$ is separable. Then $I_{\mathfrak{P}}^{Z_{f, m}}$ has exactly d orbits, each of size e, and is the stabilizer in $G_{\mathfrak{P}}^{Z_{f, m}}$ of these orbits. This implies that $G_{\mathfrak{P}}^{Z_{f, m}} / I_{\mathfrak{P}}^{Z_{f, m}} \cong \operatorname{Gal}_{k_{\mathfrak{p}}}\left(\bar{f}_{S}\right)$ as permutation groups.

Proof. By Propositions 1 and 2 both $f_{m}$ and $\widehat{f}_{m}$ are separable of degree $d e$, and $\bar{f}_{S}=\widehat{f}_{S} \bmod \mathfrak{p}$. From Statement 1 it follows that $Z_{f, m}$ is a block for $G_{\mathfrak{P}}$ and that

$$
G_{\mathfrak{P}}^{Z_{f, m}}=\operatorname{Gal}_{K_{\mathfrak{p}}}\left(\widehat{f}_{m}\right) .
$$

Now let $\widehat{L}$ be the splitting field of $\widehat{f}_{m}$ over $K_{\mathfrak{p}}$ (with $\widehat{L} \subseteq L_{\mathfrak{P}}$ ), and let $\widehat{T} \mid K_{\mathfrak{p}}$ be the maximal unramified subextension. Observe that $I_{\mathfrak{P}}$ maps onto $\operatorname{Gal}(\widehat{L} \mid \widehat{T})$ under the restriction map. The residue class field of $\widehat{T}$ is "the" splitting field of $\bar{f}_{S}$ by Statement 3 . 
By Proposition 2 and Statement 4 the $G_{\mathfrak{p}}$-orbits of $Z_{f, m}$ have length $d_{i} e$, where $d_{i}$ denotes the degree of a prime factor of $\bar{f}_{S}\left(\sum_{i} d_{i}=d\right)$. Each such orbit decomposes under $I_{\mathfrak{P}}$ into $d_{i}$ (conjugate) orbits of length $e$. We infer that $I_{\mathfrak{P}}^{Z_{f, m}}$ is the kernel of the action of $G_{\mathfrak{P}}^{Z_{f, m}}$ onto these $I_{\mathfrak{P}}$-orbits. This yields the proposition.

Proof of the Theorem. Suppose $S_{m}$ is regular and $\mathfrak{p}$ does not divide $e$. Then Proposition 3 applies. This gives assertion (i). It is now obvious that $\widehat{L}$ (notation as above) is also a splitting field for $f_{m}$. Furthermore $\operatorname{Gal}_{K_{\mathfrak{p}}}\left(f_{m}\right) \cong$ $\operatorname{Gal}_{K_{\mathfrak{p}}}\left(\widehat{f}_{m}\right)$ as permutation groups, because both agree with $\operatorname{Gal}\left(\widehat{L} \mid K_{\mathfrak{p}}\right)$ as groups and have the same degree and the same point stabilizers as permutation groups. This is (ii).

Recall that the compositum of tamely ramified extensions is tamely ramified ([9, II.7.9]). Thus from Proposition 2 we may deduce that $\widehat{L} \mid K_{\mathfrak{p}}$ is tamely ramified $(\mathfrak{p} \nmid e)$. It follows that $\widehat{L} \mid \widehat{T}$ is cyclic ([9, II.9.15]). Now statements (iii), (iv) of the Theorem are immediate consequences of Proposition 4.

7. $g$-adic Newton polygons. Suppose $S_{m}$ is not regular. Then there is a normalized $\mathfrak{p}$-integral polynomial $g \in K[X]$ whose reduction $\bmod \mathfrak{p}$ is irreducible and a multiple divisor of $f_{S} \bmod \mathfrak{p}(g \nmid f)$. Then, as a rule, we proceed by investigating the $g$-adic Newton polygon of $f$ with respect to $\mathfrak{p}$. This is defined via the $g$-adic expansion $f=\sum_{i=0}^{\widetilde{n}} \alpha_{i} g^{\widetilde{n}-i}, \widetilde{n}=[n / \operatorname{deg}(g)]$, by letting $v_{\mathfrak{p}}\left(\alpha_{i}\right)$ be the minimum of the $v_{\mathfrak{p}}$-values of the coefficients of the polynomial $\alpha_{i}=\alpha_{i}(X) \in K[X]\left(\operatorname{deg}\left(\alpha_{i}\right)<\operatorname{deg}(g)\right)$.

There is a parallel theory for $g$-adic polygons (cf. [10] and [7]). However, after passage to the unramified extension of $K_{\mathfrak{p}}$ of degree $\operatorname{deg}(g)$ we are led to expansions with regard to linear polynomials. Of course, this is most convenient (also for computations) in case $g$ itself is linear, that is, $g=X-u$ for some $\mathfrak{p}$-unit $u \in K$. Then we just have to examine the standard polygon of $\widetilde{f}(X)=f(X+u)$. Note that $\operatorname{Gal}_{K}(\widetilde{f}) \cong \mathrm{Gal}_{K}(f)$. We give some typical examples.

EXAmple 1. Let $f$ be the $p$ th cyclotomic polynomial over $K=\mathbb{Q}$ and $\mathfrak{p}=p \mathbb{Z}(n=p-1)$. The standard Newton polygon of $f$ with respect to $\mathfrak{p}$ is a side with slope 0 . Here $f \equiv(X-1)^{n}(\bmod p)$ so that we should consider the standard polygon of $\widetilde{f}(X)=f(X+1)$. It consists of one side $S=S_{m}$ with slope $m=1 / n$. So $\widetilde{f}_{S}$ is linear and $\widetilde{f}_{m}=X^{n}+p$. From the Theorem we deduce the (known) result that $\mathbb{Q}_{p}\left(\zeta_{p}\right)=\mathbb{Q}_{p}(\sqrt[p-1]{-p})$ is the $p$ th cyclotomic field over the $p$-adics.

EXAMPLE 2. Let $p$ be an odd prime and let $h \in \mathbb{Z}[X]$ be a normalized polynomial of degree $p$ which is Eisenstein with respect to $p$. Then $h$ is irreducible over $\mathbb{Q}_{p}$. Let $\pi$ be a root of $h$ and $K=\mathbb{Q}(\pi)$. Then $K_{\mathfrak{p}} \mid \mathbb{Q}_{p}$ is 
totally and wildly ramified and $v_{\mathfrak{p}}(\pi)=1, \mathfrak{p}$ being the unique prime of $K$ above $p$. Now suppose that $p^{2}$ does not divide $a=h^{\prime}(0)$ (like $h(0)$ ).

Let $f(X)=h(X) /(X-\pi)$, and let $\widetilde{f}(X)=f(X+\pi)$. This $\widetilde{f}$ is normalized of degree $n=p-1$ with $\widetilde{f}(0)=h^{\prime}(\pi)=a u$ for some principal unit $u$ of $K_{\mathfrak{p}}$. Use the fact that $p$ divides $\left(\begin{array}{l}p \\ k\end{array}\right)$ for $1 \leq k<p$. This also implies that the (standard) Newton polygon of $\tilde{f}$ is a side of length $p-1$ and height $p=v_{\mathfrak{p}}(\tilde{f}(0))$. Hence the Theorem applies (Corollary 2). In this case the Newton polygon of $f$ itself would not give any useful information. Since $u$ is a $(p-1)$ th power in $K_{\mathfrak{p}}$ we infer that $\widehat{L}=K_{\mathfrak{p}}(\sqrt[p-1]{-a})$ is a root field for $\widetilde{f}$ and $f$ and has degree $p-1$ over $K_{\mathfrak{p}}$. In particular $\left[\widehat{L}: \mathbb{Q}_{p}\right]=p(p-1)$. Since $\mathrm{Gal}_{\mathbb{Q}_{p}}(h)$ is a solvable subgroup of the symmetric group of degree $p$, a theorem of Galois now ensures that it must be the full affine group $\mathrm{AGL}_{1}(p)$ (Corollary 3.5B in [3]). It follows that $\widehat{L}=\mathbb{Q}_{p}(\pi, \sqrt[p-1]{-a})$ is the splitting field of $h$ over $\mathbb{Q}_{p}$.

It follows from the classification of the finite simple groups that, for $p \geq 5$, the affine group $\mathrm{AGL}_{1}(p)$ is a maximal subgroup of the symmetric group $\operatorname{Sym}(p)$ (see [3, p. 99 and Sect. 7.7]). Hence $\mathrm{Gal}_{\mathbb{Q}}(h)$ is either the affine group or the symmetric group.

EXAMPLE 3 . Let $n \geq 5$ be a rational prime and $b$ a rational integer not divisible by $n$. Let $f=X^{n}+a X+a$ over $K=\mathbb{Q}$ where $a=b n$. This Eisenstein trinomial has been studied by several authors. From Example 2 we know that $G=\operatorname{Gal}_{\mathbb{Q}}(f)$ is either the affine group or the symmetric group. By examining the discriminant of a root field defined by $f$ (computed in [5]) the Wegener-Hasse theorem [4] shows that $G=\operatorname{AGL}_{1}(p)$ if and only if the splitting field $L$ of $f$ contains the $n$th roots of unity (see also Movahhedi [8] for an alternative approach). Let $p$ be a prime dividing

$$
D=n^{n-1}+b(n-1)^{n-1} .
$$

This $D$ is a divisor of the discriminant of $f$, but we do not use that. Note that $D \equiv b(\bmod n)$ and that $p$ does not divide $2 b n(n-1)$. The standard polygon of $f$ with respect to $\mathfrak{p}=p \mathbb{Z}$ is a side with slope 0 .

We have $f^{\prime}=n X^{n-1}+a$ and so $n f(X)-X f^{\prime}(X)=a(n-1) X+n a$. Hence the greatest common divisor of $f$ and $f^{\prime} \bmod p$ is either 1 or $X-u \bmod p$, where $u=-n /(n-1)$ is a rational $p$-adic unit. We check that $v_{p}(f(u))=$ $v_{p}\left(f^{\prime}(u)\right)=v_{p}(D)(>0)$ and $v_{p}\left(f^{\prime \prime}(u)\right)=0$. This implies (directly) that $f \bmod p$ is inseparable. We have all the necessary information about the standard Newton polygon of $\widetilde{f}(X)=f(X+u)$ with respect to $\mathfrak{p}$ (Taylor). It consists of a side with slope 0 and length $n-2$, and a side $S_{m}$ with slope $m=v_{p}(D) / 2$ and length 2 . For the side $S_{m}$ we get $\widetilde{f}_{m}=X^{2}-c$ where

$$
c=4 u D /\left(n^{(n-1) / 2}(n-1)\right)^{2} .
$$


Note that $v_{p}(c)=v_{p}(D)$. The associated polynomial (picking $\pi=p$ ) is either $\widetilde{f}_{S}=X-c / p^{v_{p}(D)}$ or $\widetilde{f}_{S}=X^{2}-c / p^{v_{p}(D)}$, depending on whether $v_{p}(D)$ is odd or even. In any case, $\widetilde{f}_{S}$ is separable $\bmod p$, and the Theorem applies. The polynomial associated to the side with slope 0 , which agrees with its factor, must be separable $\bmod p$ as well.

If $v_{p}(D)$ is odd, $c$ is not a square in $\mathbb{Q}_{p}$. We conclude that $I_{\mathfrak{P}}$ is generated by a transposition on $Z_{\widetilde{f}}$. Since $G \cong \operatorname{Gal}_{\mathbb{Q}}(\widetilde{f})$ is a primitive permutation group (of prime degree), $G=\operatorname{Sym}(n)$ is the symmetric group by a theorem of Jordan ([3, Theorem 3.3A]).

So let $v_{p}(D)$ be even. Then $I_{\mathfrak{P}}=1$ and $G_{\mathfrak{P}}$ has either two fixed points or an orbit of length 2 on $Z_{\widetilde{f}}$, depending on whether $c$, that is, $u D$, is a square in $\mathbb{Q}_{p}$ or not. Assume $G$ is the affine group (so that $\mathbb{Q}\left(\zeta_{n}\right) \subseteq L$ ). In the first case we then must have $G_{\mathfrak{P}}=1$, because $G$ is a Frobenius group. In this case $p$ splits completely in $\mathbb{Q}\left(\zeta_{n}\right) \subseteq L$ and so $p \equiv 1(\bmod n)$. In the second case $G_{\mathfrak{P}}$ is generated by a product of $(n-1) / 2$ disjoint transpositions on $Z_{\tilde{f}}$, hence has order 2 even when restricted to $\mathbb{Q}\left(\zeta_{n}\right)$. This implies that $p \equiv-1$ $(\bmod n)$.

REMARK. It is conjectured that $G=\operatorname{Gal}_{\mathbb{Q}}\left(X^{n}+a X+a\right)$ is always the full symmetric group ( $n \geq 5$ a prime dividing the integer $a$ exactly to the first power). We have just shown that this is true if $|D|$ is not a rational square or if $D$ has a prime divisor $p \not \equiv \pm 1(\bmod n)$. Such a prime divisor is, for instance, $p=3$ if $n \equiv 2(\bmod 3)$ and $a \equiv 1(\bmod 3)$. We also have $G=\operatorname{Sym}(n)$ if $a$ is odd or if $a \equiv 2(\bmod 3)$. To see this use the fact that $X^{n}+a X+a \bmod 2$ is separable and has no root in $\mathbb{F}_{2}$ if $a$ is odd, and that the analogous statement holds mod 3 in the other case. Thus from $G=\mathrm{AGL}_{1}(n)$ it would follow that the polynomial is even irreducible mod 2 resp. 3. This would imply that $G_{\mathfrak{P}}$, for $\mathfrak{P}$ over $p=2$ resp. 3 , is the (cyclic) normal subgroup of order $n$ in $G$ and that 2 or 3 split completely in $\mathbb{Q}\left(\zeta_{n}\right) \subseteq L$, which is impossible.

One also knows that $G=\operatorname{Sym}(n)$ if $D<0$ or, more generally, if $a<n^{2}$. No example is known so far where $G \neq \operatorname{Sym}(n)$.

\section{References}

[1] E. Brieskorn und H. Knörrer, Ebene algebraische Kurven, Birkhäuser, Basel, 1981.

[2] S. D. Cohen, A. Movahhedi and A. Salinier, Double transitivity of Galois groups of trinomials, Acta Arith. 82 (1997), 1-15.

[3] J. D. Dixon and B. Mortimer, Permutation Groups, Springer, Berlin, 1996.

[4] H. Hasse, Über die Diskriminante auflösbarer Körper von Primzahlgrad, J. Reine Angew. Math. 176 (1936), 12-17. 
[5] P. Llorente, E. Nart and N. Vila, Discriminants of number fields defined by trinomials, Acta Arith. 43 (1984), 367-373.

[6] B. H. Matzat, Konstruktive Galoistheorie, Lecture Notes in Math. 1284, Springer, 1987.

[7] J. Montes and E. Nart, On a theorem of Ore, J. Algebra 146 (1992), 318-334.

[8] A. Movahhedi, Galois group of $X^{p}+a X+a$, ibid. 180 (1996), 966-975.

[9] J. Neukirch, Algebraische Zahlentheorie, Springer, Berlin, 1992.

[10] Ö. Ore, Newtonsche Polygone in der Theorie der algebraischen Körper, Math. Ann. 99 (1928), 84-117.

[11] B. L. van der Waerden, Die Zerlegungs- und Trägheitsgruppe als Permutationsgruppen, ibid. 111 (1937), 731-733.

Mathematisches Institut

Universität Tübingen

Auf der Morgenstelle 10

D-72076 Tübingen, Germany

E-mail: peter.schmid@uni-tuebingen.de 